\title{
Measuring Proton Shift Tensors with Ultrafast MAS NMR
}

\author{
Habeeba K. Miah†, David A. Bennett †, Dinu Iuga ${ }^{\ddagger}$, and Jeremy J. Titman ${ }^{\star *}$ \\ tSchool of Chemistry, University of Nottingham, University Park, Nottingham, NG7 2RD, UK \\ ‡UK 850 MHz Solid-state NMR Facility, Department of Physics, Millburn House, University of Warwick, Coventry
}

CV4 7AL, UK

*Corresponding author: Email: Jeremy.Titman@nottingham.ac.uk, Tel: +44 1159513560

\begin{abstract}
A new proton anisotropic-isotropic shift correlation experiment is described which operates with ultrafast MAS, resulting in good resolution of isotropic proton shifts in the detection dimension. The new experiment makes use of a recoupling sequence designed using symmetry principles which reintroduces the proton chemical shift anisotropy in the indirect dimension. The experiment has been used to measure the proton shift tensor parameters for the $\mathrm{OH}$ hydrogen-bonded protons in tyrosine. $\mathrm{HCl}$ and citric acid at Larmor frequencies of up to $850 \mathrm{MHz}$.
\end{abstract}

Keywords: chemical shift anisotropy, ultrafast magic angle spinning, anisotropic-isotropic shift correlation, hydrogen bonding, symmetry-based recoupling sequence

\section{Introduction}

Hydrogen bonding plays a critical role in directing molecular self assembly, which is ubiquitous in biology and an important aspect of supramolecular chemistry, crystal engineering and nanotechnology. Solid-state ${ }^{1} \mathrm{H}$ NMR spectroscopy provides an excellent means to study hydrogen-bonded structures, because the ${ }^{1} \mathrm{H}$ chemical shift interaction contains information about the geometry of the hydrogen bond. For example, an early study [1] revealed a relationship between the ${ }^{1} \mathrm{H}$ isotropic shift $\delta_{\text {iso }}$ and the O...O distance in a broad range of crystalline solids forming O-H...O hydrogen bonds. By comparison with neutron diffraction data, which provides accurate positions for the hydrogen atoms, a linear correlation was later established between $\delta_{\text {iso }}$ and the H...O hydrogen bond length [2]. As confirmed by ab initio calculations [3], the main origin of this effect is the deshielding of the principal component of the ${ }^{1} \mathrm{H}$ chemical shift tensor perpendicular to the hydrogen bond that occurs as the bond length decreases. More recently, Wu et al. [4] showed for a series of crystalline hydrates that the span and the skew of the ${ }^{1} \mathrm{H}$ chemical shift tensor were linearly correlated to the 
hydrogen bond length and the O-H...O bond angle, respectively. A similar correlation was identified between calculated values of the ${ }^{1} \mathrm{H}$ chemical shift anisotropy (CSA) and $\mathrm{H} . . . \mathrm{O}$ distances in galactose [5]. These results suggest that variations in individual principal components are more sensitive indicators of changes in the hydrogen bond environment than the ${ }^{1} \mathrm{H}$ isotropic shift alone.

However, measurements of the ${ }^{1} \mathrm{H}$ shift tensor in solids are challenging, because of strong ${ }^{1} \mathrm{H}$ homonuclear dipolar couplings, which broaden the lines and reduce resolution, and the relatively small size of the ${ }^{1} \mathrm{H}$ chemical shift interaction. Until recently, experiments have been restricted to simple systems, usually in the form of single crystals [6-9] or perdeuterated powders [4], and the resulting lack of experimental data has precluded a full understanding of the relationship between ${ }^{1} \mathrm{H}$ shift tensors and hydrogen bond geometry. However, some progress has now been made towards more general methodologies for measuring the ${ }^{1} \mathrm{H}$ shift tensor based on two-dimensional NMR experiments which correlate the anisotropic and isotropic parts of the shift interaction. These make use of magic angle spinning (MAS) and multi-pulse homonuclear decoupling $[10,11]$ to resolve different ${ }^{1} \mathrm{H}$ sites via their isotropic shifts in the detection dimension, so that a method of reintroducing or "recoupling" the MAS-averaged ${ }^{1} \mathrm{H}$ CSA is required during the evolution time. For example, Brouwer and Ripmeester [12] designed a recoupling sequence using symmetry principles $[13,14]$, while Duma et al. [15] used rotary resonance [16-18] to reintroduce the ${ }^{1} \mathrm{H}$ CSA. In common with all methods based on rotary resonance, the latter suffers from substantial sensitivity to rf inhomogeneity, while the sequence symmetry chosen in the former restricts the experiment to relatively slow MAS rates. In order to improve the resolution of different sites in $v_{2}$, Hou et al. [19] recently exploited magnetization transfer to a neighbouring heteronucleus after recoupling the ${ }^{1} \mathrm{H}$ shift anisotropy during $t_{1}$. This approach was shown to be particularly powerful for measuring the ${ }^{1} \mathrm{H}$ shift tensor parameters for the amide protons in ${ }^{15} \mathrm{~N}$-enriched proteins, but is less appropriate for hydrogen-bonded $\mathrm{OH}$ sites where a suitable directly bonded heteronucleus is not available.

In this communication, we demonstrate a ${ }^{1} \mathrm{H}$ anisotropic-isotropic shift correlation experiment which employs a symmetry-based recoupling sequence which is suitable for use when the MAS rate exceeds 50 $\mathrm{kHz}$. The combination of MAS in this "ultrafast" regime and high $\mathrm{B}_{0}$ field allows the majority of hydrogenbonded sites in simple crystalline solids to be resolved in $v_{2}$ without the need for multi-pulse homonuclear decoupling. The usefulness of the new experiment is demonstrated by measurements of the ${ }^{1} \mathrm{H}$ shift tensors for all the hydrogen-bonded $\mathrm{OH}$ sites in tyrosine. $\mathrm{HCl}$ and citric acid. 


\section{Pulse Sequence Design}

Levitt and co-workers $[13,14]$ have used symmetry principles to design recoupling sequences which can reintroduce specific nuclear spin interactions averaged by MAS. The current work employs the most flexible class of symmetry-based recoupling sequence which is designated $R N_{n^{r}}$. These consist of $\mathrm{N}$ composite inversion pulses $\mathrm{R}$, timed to occupy $n$ rotor periods, where each $\mathrm{R}$ element of the overall sequence has duration $n \tau_{\mathrm{r}} / N$ and alternate elements have phases $\pm \pi n v / N$. It has been demonstrated that the symmetry numbers $N, n$ and $v$ determine which interactions are retained in the first-order effective Hamiltonian for the sequence according to:

$$
H^{(1)} \propto A_{m}^{l} T_{\mu}^{\lambda} \quad \text { if }(n m-v \mu)=\frac{N}{2} k_{\lambda}
$$

where $A$ is a rank- $l$ irreducible spherical tensor operator describing the spatial part of the interaction Hamiltonian, and $\mathrm{T}$ is a rank- $\lambda$ irreducible spherical tensor operator describing the spin part. The rotational components of the spatial and spin tensors $m$ and $\mu$ take values $m=l, l-1, \ldots-l$ and $\mu=\lambda, \lambda-1, \ldots-\lambda$, respectively, and $k_{\star}$ is any integer with the same parity as $\lambda$.

Recoupling sequences suitable for measuring the ${ }^{1} \mathrm{H}$ shift tensor recouple a single-quantum Hamiltonian which takes the form

$$
H^{(1)}=\sum_{j}\left(\omega_{j} T_{-1}^{1}+\omega_{j}^{*} T_{1}^{1}\right)
$$

to first order, where the index $j$ runs over all the ${ }^{1} \mathrm{H}$ shift interactions. The coefficient $\omega_{j}$ depends on the anisotropic part of the chemical shift interaction and determines the appearance of the recoupled powder pattern observed in the evolution dimension. Suitable symmetries must also avoid inadvertently recoupling the strong ${ }^{1} \mathrm{H}$ dipolar interactions, as well as isotropic ${ }^{1} \mathrm{H}$ shifts. Of the many symmetries which satisfy these conditions, $\mathrm{R} 18_{2}^{5}[12]$ and $\mathrm{R} 12_{1}^{4}[19]$ have been used successfully to measure ${ }^{1} \mathrm{H}$ shift tensor parameters. However, since the pulse sequence is synchronized with the spinning, the required $\mathrm{rf}$ amplitude increases with $\omega_{r}$, so that these symmetries become impractical at ultrafast MAS rates. For $\mathrm{R} 18_{2}^{5}$ and $\mathrm{R} 12_{1}^{4} \mathrm{rf}$ amplitudes in excess of $180 \mathrm{kHz}$ and $240 \mathrm{kHz}$, respectively, would be required for MAS rates above $40 \mathrm{kHz}$, and in Refs. [12] and [19] experiments were carried out below $20 \mathrm{kHz}$. A different choice of sequence symmetry allows similar experiments to be carried out at higher MAS rates at which a well-resolved isotropic ${ }^{1} \mathrm{H}$ spectrum can be acquired without the need for homonuclear decoupling as in Ref. [12]. Note 
that in the case of $\mathrm{OH}$ protons, resolution of different sites cannot be improved by magnetization transfer to a directly bonded heteronucleus as in Ref. [19].

The pulse sequence used to record ${ }^{1} \mathrm{H}$ anisotropic-isotropic shift correlation spectra in this work is shown in Figure 1. The preparation period consists only of a suitable relaxation delay, which is followed immediately by a $\mathrm{R} 16_{3}^{2}$ recoupling sequence of duration $t_{1}$. This choice of $\mathrm{R}$ sequence symmetry allows MAS rates up to $67.5 \mathrm{kHz}$ to be used before the required rf amplitude exceeds $180 \mathrm{kHz}$. During the evolution period, longitudinal ${ }^{1} \mathrm{H}$ magnetization decays under the action of the Hamiltonian in Eq. (2), and subsequently a $\pi / 2$ pulse transfers any remaining longitudinal ${ }^{1} \mathrm{H}$ magnetization to the transverse plane where it is acquired during $t_{2}$. In practice, the experiment is initiated with a saturation sequence followed by a recovery delay to ensure constant initial magnetization and to avoid long recycle delays.

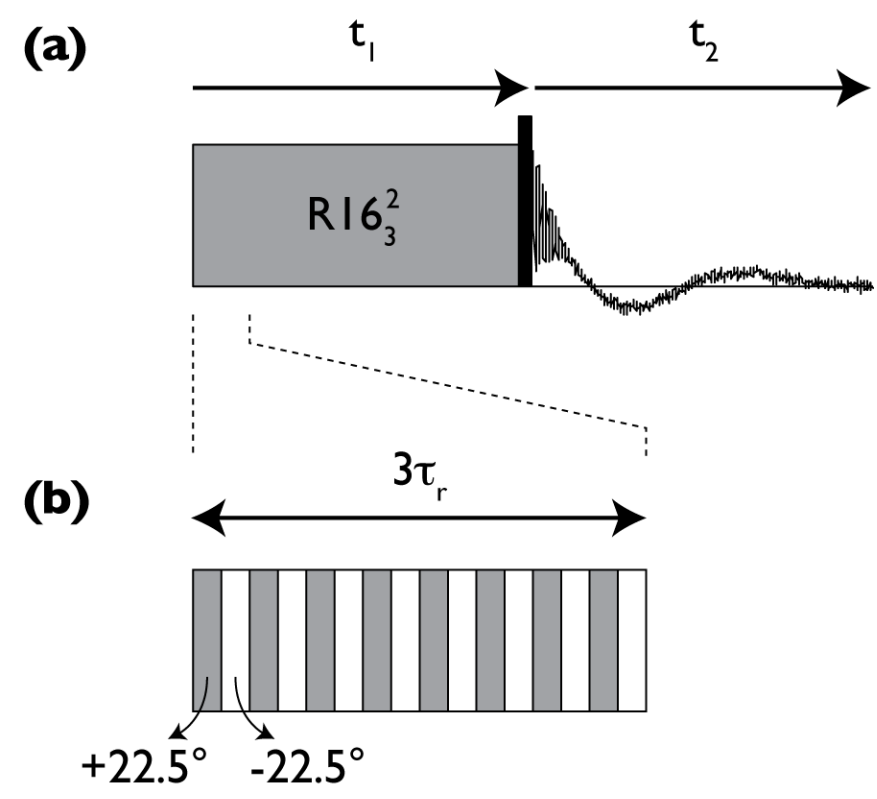

Figure 1. Experiment used to record ${ }^{1} \mathrm{H}$ anisotropic-isotropic shift correlation spectra at ultrafast MAS rates, as described in the text: (a) overall pulse sequence. The black bar represents a $\pi / 2$ pulse. (b) R16 ${ }_{3}^{2}$ sequence which operates during the evolution period, $t_{1}$. Each bar represents a $\pi$ pulse.

\section{Experimental}

${ }^{1} \mathrm{H}$ anisotropic-isotropic shift correlation spectra were recorded using the pulse sequence shown in Figure 1 on two spectrometers operating at Larmor frequencies of 600.13 and $850.13 \mathrm{MHz}$, both equipped with a 1.3 mm MAS probe. MAS rates of between 58.6 and $62.5 \mathrm{kHz}$ were selected, so that the $\mathrm{R} 16_{3}^{2}$ recoupling sequence required a ${ }^{1} \mathrm{H} \mathrm{rf}$ amplitude of either 156.3 or $166.6 \mathrm{kHz}$. Note that the small coil diameter means that these amplitudes are well inside the manufacturer's specifications for the ultrafast MAS probes used 
here. Further experimental details are given in the relevant figure captions. The two-dimensional FID was Fourier transformed with respect to $t_{2}$, and the resulting spectrum was baseline corrected to remove the broad ${ }^{1} \mathrm{H}$ background signal. Since $t_{1}$ samples the decay of longitudinal ${ }^{1} \mathrm{H}$ magnetization quadrature detection is not appropriate, and a real Fourier transform was applied in the indirect dimension, resulting in ${ }^{1} \mathrm{H}$ CSA lineshapes in $v_{1}$ which are symmetrical about the origin. For anisotropic interactions, such as the CSA, the magnitude of the recoupled Hamiltonian always depends on the orientation of the molecular frame with respect to the rotor. This means that not all orientations are fully recoupled, and in this case there is some longitudinal ${ }^{1} \mathrm{H}$ magnetization which does not decay, resulting in a dc offset in the $t_{1}$ signal and a corresponding zero-frequency line in $v_{1}$. This was removed by subtracting the average of the final 8 points of each interferogram prior to Fourier transform in $v_{1}$. A schematic describing all the processing steps is included in the Supplementary Information (Figure S1). The $t_{1}$ dwell time must correspond to an even number of $\pi$ pulses, but if sampling does not coincide with a complete $\mathrm{R}$ cycle, cycling sidebands appear in the $v_{1}$ dimension. Hence, a careful choice of sampling rate in the indirect dimension is necessary to ensure that these appear outside the ${ }^{1} \mathrm{H}$ CSA lineshape.

The anisotropy and asymmetry parameters for the chemical shift are defined according to $\zeta=\delta_{z z}-\delta_{i s o}$ and $\eta$ $=\left(\delta_{y y}-\delta_{x x}\right) / \zeta$, respectively, with the principal components ordered according to $\left|\delta_{z z}-\delta_{\text {iso }}\right| \geq\left|\delta_{x x}-\delta_{i s o}\right| \geq$ $\left|\delta_{y y}-\delta_{i s o}\right|$. Numerical simulations of the recoupled ${ }^{1} \mathrm{H}$ CSA lineshapes were performed using SIMPSON [20]. Powder averaging was achieved using $615(\alpha, \beta)$ orientations chosen according to the scheme of Zaremba [21] and 40 uniformly distributed values of $\gamma$. $B_{1}$ inhomogeneity was included by summing 17 simulations carried out using $\mathrm{rf}$ amplitudes weighted according to the experimentally determined $B_{1}$ distribution. The experimental distribution is skewed with its maximum on the high $B_{1}$ side and a long tail extending on the low side with significant intensity limited to $\pm 5 \%$ of the nominal value. A Gaussian line broadening function was applied and the result processed in an identical fashion to the experimental data in order to obtain a simulated ${ }^{1} \mathrm{H}$ CSA lineshape. An array of these simulated lineshapes were generated for $\zeta$ up to $25 \mathrm{ppm}$ and $\eta$ in the range 0.0 to 1.0 , and the ${ }^{1} \mathrm{H}$ shift tensor parameters were extracted by comparing these with the experimental data, using a similar method to that described in Ref. [12]. For each simulated lineshape the optimal scaling factor was found by fitting to the experimental data, and the corresponding $\chi^{2}$ parameter was plotted as a function of $\zeta$ and $\eta$. The resulting error surface allows the best-fit values of $\zeta$ and $\eta$ to be obtained, as well as their confidence limits, assuming $\chi^{2}$ is suitably normalized [22]. 


\section{Results and Discussion}

Figure 2 shows SIMPSON simulations performed as described above of ${ }^{1} \mathrm{H}$ CSA lineshapes recoupled using a $\mathrm{R} 6_{3}^{2}$ sequence operating at a MAS rate of $62.5 \mathrm{kHz}$ for a Larmor frequency of $600 \mathrm{MHz}$. These simulations demonstrate that measurement of the chemical shift parameters $\zeta$ and $\eta$ is possible using the anisotropicisotropic correlation spectrum described here. In (a) the increasing $\zeta$ results in an increase in the width of the pattern, while the sensitivity of the lineshape to $\eta$ is demonstrated in (b). The experimentally determined $B_{1}$ distribution has been added to otherwise identical simulations which are shown in (c) and (d). Changing the $\mathrm{R}$ symmetry results in a different scaling factor, so the lineshapes observed with $\mathrm{R} 16_{3}^{2}$ are similar but not identical to those for $\mathrm{R} 18_{2}^{5}$ [12] or $\mathrm{R} 12_{1}^{4}$ [19]. Even at the relatively low field of $600 \mathrm{MHz}$, values of $\xi$ below 5 ppm can be determined as illustrated in (a). In practice the variation with $\eta$ seen in (b) is obscured to some extent by effect of $B_{1}$ inhomogeneity, as illustrated in (d). In common with $R 18_{2}^{5}$ [12] or R12 ${ }_{1}^{4}$ [19], the sign of $\zeta$ cannot be determined because of the symmetry of the recoupled ${ }^{1} \mathrm{H}$ CSA lineshape. Furthermore, $\mathrm{R}$ sequences with this symmetry also reintroduce the heteronuclear dipolar interaction, so they are not suitable for measuring ${ }^{1} \mathrm{H}$ CSAs in the presence of abundant nuclei without additional decoupling [19]. 

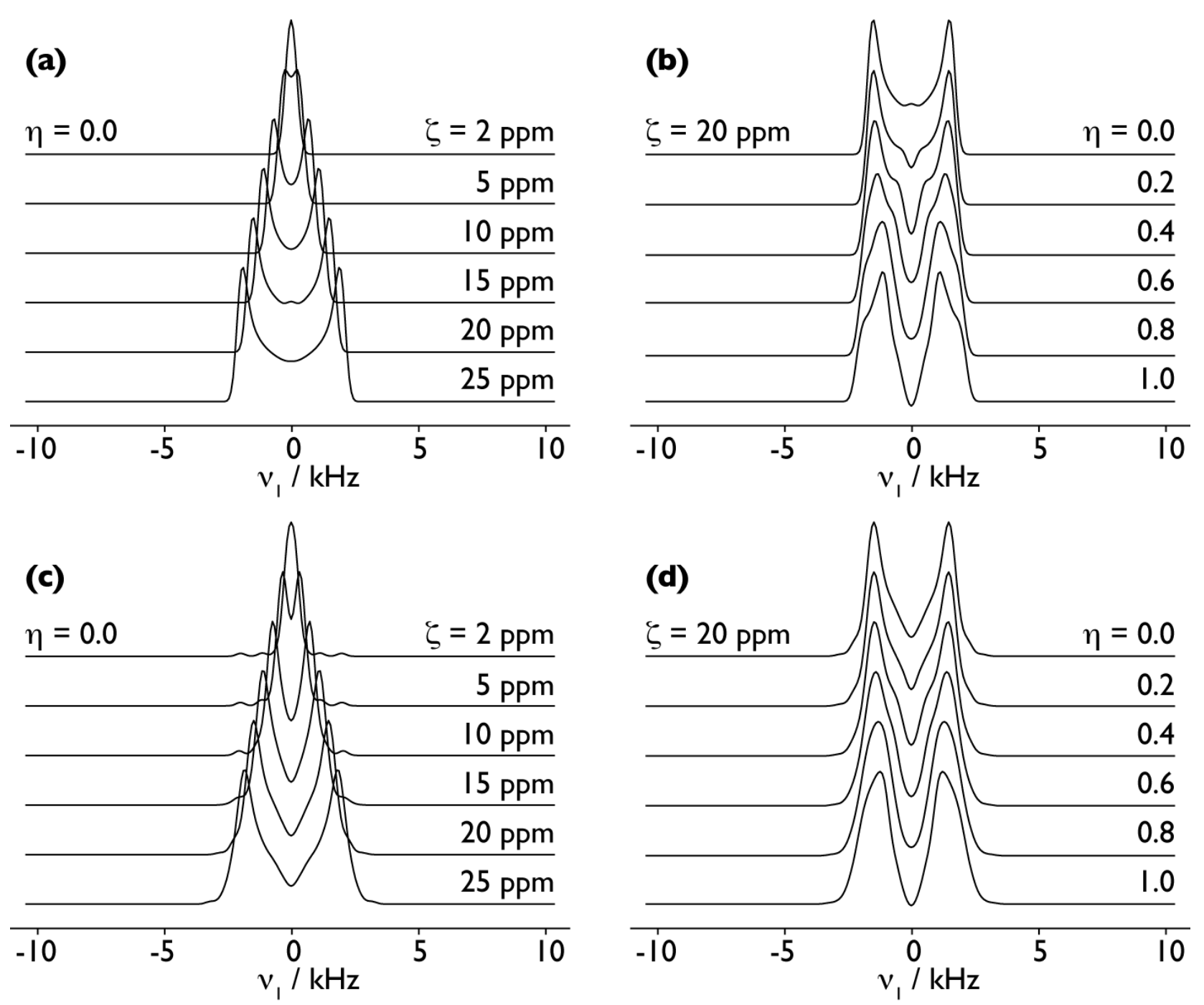

Figure 2. SIMPSON simulations of ${ }^{1} \mathrm{H}$ CSA lineshapes recoupled using a $\mathrm{R} 16_{3}^{2}$ sequence for a Larmor frequency of $600 \mathrm{MHz}$ and a MAS rate of $62.5 \mathrm{kHz}$, carried out as described in the text. The simulations in (a) and (c) show the variation of the recoupled lineshape with $\zeta$ for $\eta=0.0$, while (b) and (d) show the variation with $\eta$ for $\xi=20 \mathrm{ppm}$. The effects of $B_{1}$ inhomogeneity are neglected in (a) and (b), while the experimentally determined $B_{1}$ distribution is included in (c) and (d). Note the broadening of the singularities in the latter as a result of the $B_{1}$ inhomogeneity, and the consequent loss of sensitivity to $\eta$ in particular.

As an illustration of the method Figure 3(a) shows a ${ }^{1} \mathrm{H}$ anisotropic-isotropic correlation spectrum of tyrosine. $\mathrm{HCl}$ recorded at a Larmor frequency of $850.13 \mathrm{MHz}$ and a MAS rate of $58.6 \mathrm{kHz}$. Other experimental parameters are given in the caption. The high-field ultrafast MAS ${ }^{1} \mathrm{H}$ spectrum (top) exhibits two well-resolved peaks which have been assigned previously by measurement of ${ }^{1} \mathrm{H}-{ }^{17} \mathrm{O}$ internuclear distances using heteronuclear recoupling experiments [23]. The neutron structure of tyrosine. $\mathrm{HCl}$ [24] contains two short hydrogen bonds both involving the phenolic $\mathrm{OH}$ (designated $\mathrm{O} \cdot \mathrm{H}$ " in Ref. [24]) which acts 
as the acceptor group in a hydrogen bond with the carboxylic $\mathrm{OH}\left(\right.$ designated $\mathrm{O}^{2} \mathrm{H}^{2}$ ) and simultaneously as the donor in a hydrogen bond with the chloride ion. Figure 3(b) shows (red lines) cross-sections parallel to $v_{1}$ taken at $v_{2}$ frequencies corresponding to the isotropic ${ }^{1} \mathrm{H}$ shifts of $\mathrm{H}^{2}$ and $\mathrm{H}^{n}$. These are compared with SIMPSON simulations (dashed lines) for the best-fit $\zeta$ and $\eta$ obtained as described above. The results of the fits are given in Table 1, and the error surfaces with respect to $\zeta$ and $\eta$ are shown in the Supplementary Information (Figure S2). The sensitivity to $\eta$ evident in Figure 2(b) is substantially reduced in experimental $v_{1}$ cross-sections due to $\mathrm{rf}$ inhomogeneity which broadens the ${ }^{1} \mathrm{H}$ CSA lineshape. Omitting rf inhomogeneity from the simulations used to extract the ${ }^{1} \mathrm{H}$ shift parameters reduces the quality of the fit and results in the overestimation of $\eta$.
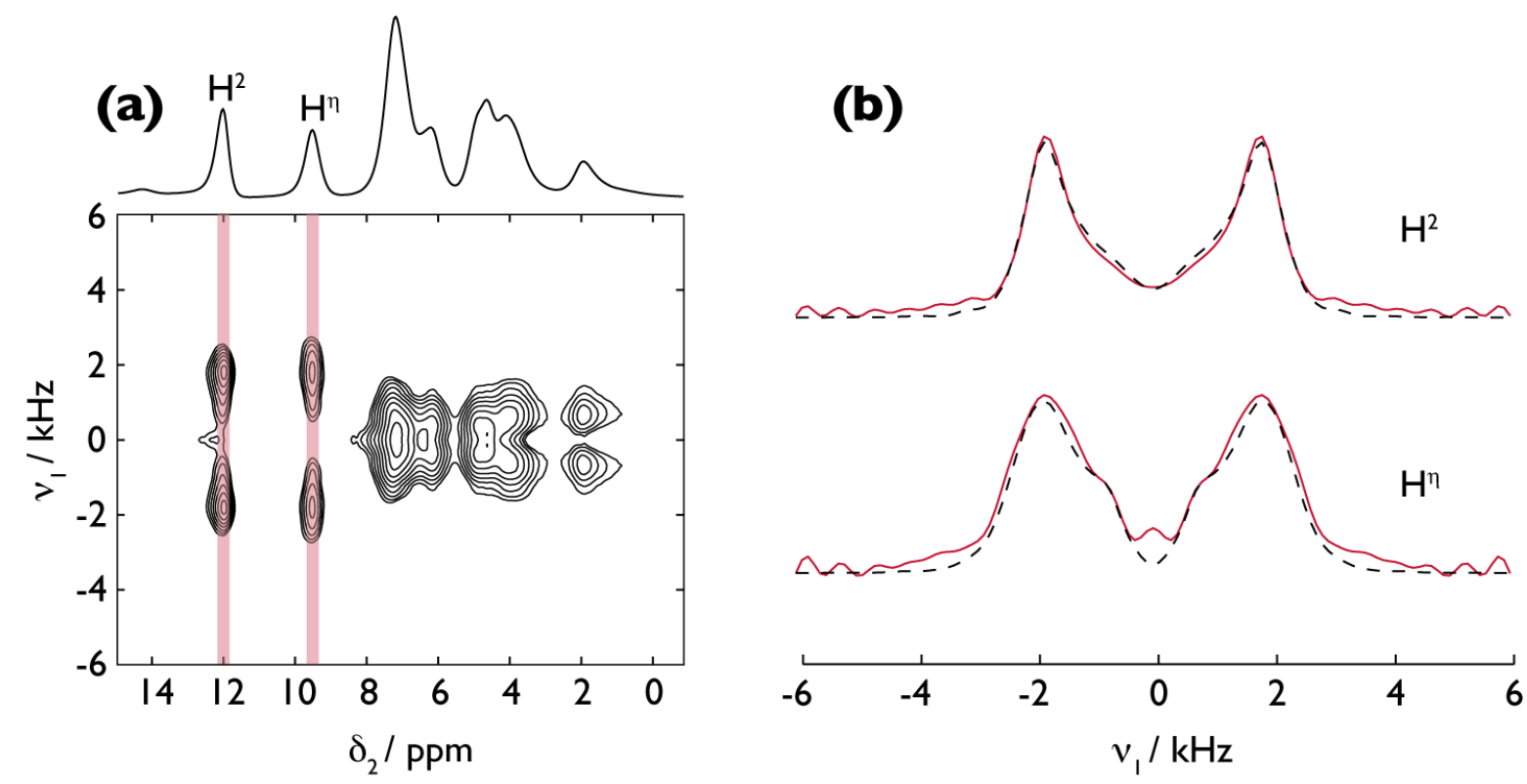

Figure 3. (a) shows a ${ }^{1} \mathrm{H}$ anisotropic-isotropic correlation spectrum of tyrosine. $\mathrm{HCl}$ recorded at a Larmor frequency of $850.13 \mathrm{MHz}$. The MAS rate was $58.6 \mathrm{kHz}$, so that the $\mathrm{R} 16_{3}^{2}$ recoupling sequence required a ${ }^{1} \mathrm{H}$ rf amplitude of $156.3 \mathrm{kHz}$, corresponding to a $\pi$ pulse duration of $3.2 \mu$ s. There were $48 t_{1}$ increments with data points sampled every $12 \mathrm{R}$ elements, resulting in a dwell time in the indirect dimension of $38.4 \mu \mathrm{s}$. In $t_{2}$ 2048 complex points were acquired with a spectral width of $150 \mathrm{kHz}$. Saturation was achieved using a train of $200 \pi / 2$ pulses separated by intervals of $10 \mathrm{~ms}$ with a recovery delay of $4 \mathrm{~s} .{ }^{1} \mathrm{H}$ chemical shifts were referenced to $\mathrm{Me}_{4} \mathrm{Si}$, and the assignments of the ${ }^{1} \mathrm{H}$ sites are taken from Ref. [23] using the labelling scheme from Ref. [24]. (b) shows (red lines) cross-sections parallel to $v_{1}$ taken at $v_{2}$ frequencies corresponding to the isotropic ${ }^{1} \mathrm{H}$ shifts of the hydrogen-bonded $\mathrm{OH}$ protons $\mathrm{H}^{2}$ and $\mathrm{H}^{n}$, along with SIMPSON simulations (dashed lines) for the best-fit chemical shift parameters. 
As a further test Figure 4(a) shows a ${ }^{1} \mathrm{H}$ anisotropic-isotropic correlation spectrum of anhydrous citric acid recorded at a lower Larmor frequency of $600.13 \mathrm{MHz}$ and a MAS rate of $62.5 \mathrm{kHz}$. Other experimental parameters are given in the caption. The ultrafast MAS ${ }^{1} \mathrm{H}$ spectrum (top) exhibits four well-resolved peaks which have been partially assigned in Ref. [12] by comparison with the single-crystal X-ray structure [25]. Figure 4(b) shows (red lines) cross-sections parallel to $v_{1}$ taken at $v_{2}$ frequencies corresponding to the isotropic ${ }^{1} \mathrm{H}$ shifts of the four hydrogen-bonded sites. These are compared with SIMPSON simulations (dashed lines) for the best-fit $\zeta$ and $\eta$ parameters. The results of the fits are given in Table 1 , and the error surfaces are shown in the Supplementary Information (Figure S3). There is generally good agreement with the $\zeta$ values measured by Brouwer and Ripmeester at higher $\mathrm{B}_{0}$ field and lower MAS rate [12]. Ultrafast MAS causes sample heating which might cause partial averaging of the ${ }^{1} \mathrm{H}$ CSA, but there is no systematic difference between the values obtained at different MAS rates which would suggest this is a problem here. Finally, the effect of $\mathrm{rf}$ inhomogeneity results in a fairly significant uncertainty for $\eta$, but once again this is comparable to that obtained at lower MAS rates.

(a)

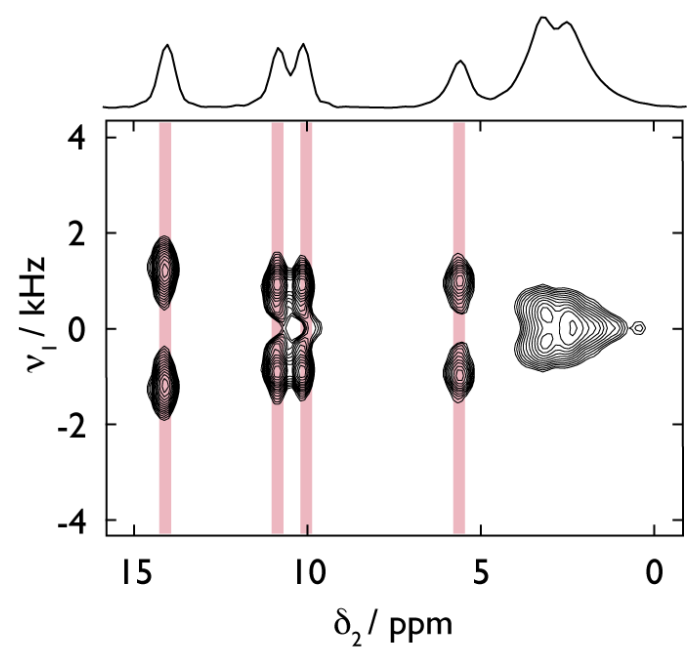

(b)

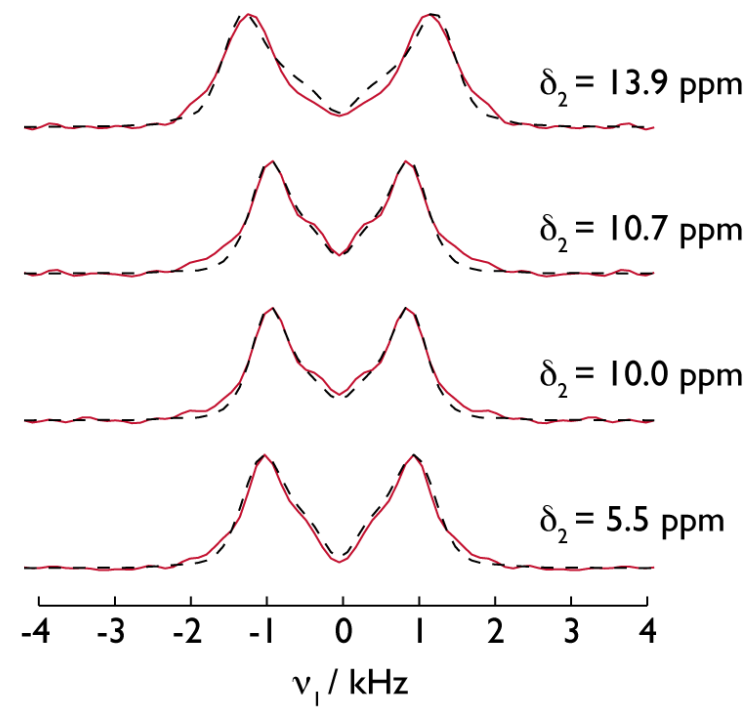

Figure 4. (a) shows a ${ }^{1} \mathrm{H}$ anisotropic-isotropic correlation spectrum of anhydrous citric acid recorded at a Larmor frequency of $600.13 \mathrm{MHz}$. The MAS rate was $62.5 \mathrm{kHz}$, so that the $\mathrm{R} 16_{3}^{2}$ recoupling sequence required a ${ }^{1} \mathrm{H}$ rf amplitude of $166.6 \mathrm{kHz}$, corresponding to a $\pi$ pulse duration of $3.0 \mu \mathrm{s}$ and a dwell time in $\mathrm{t}_{1}$ of $36.0 \mu \mathrm{s}$. Other parameters were identical to those given in the caption to Figure 3, except that the recovery delay was $120 \mathrm{~s}$. (b) shows (red lines) cross-sections parallel to $v_{1}$ taken at $v_{2}$ frequencies corresponding to the isotropic ${ }^{1} \mathrm{H}$ shifts of the hydrogen-bonded $\mathrm{OH}$ protons, along with SIMPSON simulations (dashed lines) for the best-fit chemical shift parameters. 
Table 1. Values of ${ }^{1} \mathrm{H}$ chemical shift parameters (with $95 \%$ confidence limits) measured in this work from fitting to simulated spectra as described in the text.

\begin{tabular}{||c|c|c|c||}
\hline \hline Site & $\delta_{\text {iso }}$ & $\xi$ & $\eta$ \\
\hline \hline \multicolumn{4}{|c||}{ Tyrosine.HCl $^{\mathrm{a}}$} \\
\hline $\mathrm{H}^{2}$ & 12.0 & $17.5(17.3,17.7)$ & $0.0(0.0,0.1)$ \\
\hline $\mathrm{H}^{n}$ & 9.6 & $18.5(18.0,19.0)$ & $0.5(0.4,0.7)$ \\
\hline \multicolumn{4}{|c|}{ Citric acid $^{\mathrm{b}}$} \\
\hline $\mathrm{H}^{5}$ & 13.9 & $16.9(16.5,17.3)$ & $0.3(0.0,0.5)$ \\
\hline $\mathrm{H}^{6,7 \mathrm{c}}$ & 10.7 & $12.5(12.2,12.8)$ & $0.3(0.0,0.4)$ \\
\hline $\mathrm{H}^{6,7 \mathrm{c}}$ & 10.0 & $12.5(12.2,13.8)$ & $0.2(0.0,0.4)$ \\
\hline $\mathrm{H}^{8}$ & 5.5 & $13.6(13.5,14.2)$ & $0.4(0.0,0.7)$ \\
\hline \hline
\end{tabular}

a. ${ }^{1} \mathrm{H}$ sites labelled according to Ref. [24]

b. ${ }^{1} \mathrm{H}$ sites labelled according to Ref. [25]

c. sites $\mathrm{H}^{6}$ and $\mathrm{H}^{7}$ cannot be assigned.

\section{Conclusion}

A new ${ }^{1} \mathrm{H}$ anisotropic-isotropic shift correlation experiment has been described which operates with ultrafast MAS, resulting in good resolution of isotropic ${ }^{1} \mathrm{H}$ shifts in the detection dimension, especially in combination with high $B_{0}$ fields. A recoupling sequence is required to reintroduce the ${ }^{1} \mathrm{H}$ CSA in the indirect dimension, but the symmetry numbers must be carefully chosen in order to avoid high rf amplitudes, and the R16 ${ }_{3}^{2}$ sequence was found to be an appropriate choice for MAS rates over $60 \mathrm{kHz}$. The new experiment has been used to measure the ${ }^{1} \mathrm{H}$ shift tensor for all the hydrogen-bonded $\mathrm{OH}$ sites in tyrosine. $\mathrm{HCl}$ and citric acid. Good agreement was found between the resulting values of $\xi$ and $\eta$ and those obtained previously. A number of possible improvements to the experiment can be envisioned. The ${ }^{1} \mathrm{H}$ CSA could be recoupled using a modified version of the ROCSA experiment due to Chan and Tycko which has been used to measure ${ }^{13} \mathrm{C}$ shift parameters in uniformly labelled amyloid fibrils [26]. This approach has the advantage that the resulting undistorted ${ }^{1} \mathrm{H}$ CSA lineshapes allow measurement of the sign of $\zeta$. The effect of $B_{1}$ inhomogeneity could be reduced by employing amplitude- or phase-modulated R elements after the fashion of Nishiyama et al. [27] Alternatively, the signal which results from the extremes of the $B_{1}$ distribution could be filtered out by extending the duration of the $\pi / 2$ magnetization transfer pulse, as described in a different context by $\mathrm{Lu}$ et al. [28] These modifications are currently under investigation in our laboratory. 


\section{Acknowledgments}

HM and DB thank the EPSRC and the University of Nottingham for PhD studentships. The UK $850 \mathrm{MHz}$ solid-state NMR Facility used in this research was funded by EPSRC and BBSRC, as well as the University of Warwick, including part funding through Birmingham Science City Advanced Materials Projects 1 and 2, supported by Advantage West Midlands (AWM) and the European Regional Development Fund (ERDF).

\section{References}

[1] B. Berglund, R. W. Vaughan, Correlations between proton chemical shift tensors, deuterium quadrupole couplings, and bond distances for hydrogen bonds in solids, J. Chem. Phys., 73 (1980) 2037-2043.

[2] G. A. Jeffrey, Y. Yeon, The correlation between hydrogen-bond lengths and proton chemical shifts in crystals, Acta Crystallogr. B, 42 (1986) 410-413.

[3] C. M. Rohlfing, L. C. Allen, R. Ditchfield, Proton chemical shift tensors in hydrogen-bonded dimers of RCOOH and ROH, J. Chem. Phys., 79 (1983) 4958-4966.

[4] G. $\mathrm{Wu}, \mathrm{C}$. J. Freure, E. Verdurand, Proton chemical shift tensors and hydrogen bond geometry: a ${ }^{1} \mathrm{H}^{-}$ ${ }^{2} \mathrm{H}$ dipolar NMR study of the water molecule in crystalline hydrates, J. Am. Chem. Soc., 120 (1998) 1318713193.

[5] M. Kibalchenko, D. Lee, L. Shao, M. C. Payne, J. J. Titman, J. R. Yates, Distinguishing hydrogen bonding networks in $\alpha$-D-galactose using NMR experiments and first principles calculations, Chem. Phys. Lett., 498 (2010) 270-276.

[6] W. K. Rhim, D. D. Elleman, R. W. Vaughan, J. Chem. Phys., Analysis of multiple pulse NMR in solids, 59 (1973) 3740-3749.

[7] P. Van Hecke, J. C. Weaver, B. L. Neff, J. S. Waugh, Determination of the proton chemical shielding tensor in anhydrous $\alpha$-oxalic acid by multiple pulse NMR, J. Chem. Phys., 60 (1974) 1668-1670.

[8] R. Grosescu, A. M. Achlama, U. Haberlen, H. W. Spiess, Multiple pulse study of proton shielding in single crystals of maleic acid, Chem. Phys., 5 (1974) 119-128.

[9] U. Burghoff, G. Scheler, R. Muller, High-resolution proton magnetic resonance investigations of $\mathrm{KH}_{2} \mathrm{PO}_{4}$ single-crystals by simultaneous phosphorus spin-decoupling, Phys. Status Solidi A, 25 (1974) K31K33.

[10] E. Vinogradov, P. Madhu, S. Vega, High-resolution proton solid-state NMR spectroscopy by a phasemodulated Lee-Goldburg experiment, Chem. Phys. Lett., 314 (1999) 443-450.

[11] D. Sakellariou, A. Lesage, P. Hodgkinson, L. Emsley, Homonuclear dipolar decoupling in solid-state NMR using continuous phase modulation, Chem. Phys. Lett., 319 (2000) 253-260.

[12] D. H. Brouwer, J. A. Ripmeester, Symmetry-based recoupling of proton chemical shift anisotropies in ultrahigh-field solid-state NMR, J. Magn. Reson., 185 (2007) 173-178.

[13] M. Carravetta, M. Eden, X. Zhao, A. Brinkmann, M. H. Levitt, Symmetry principles for the design of radiofrequency pulse sequences in the nuclear magnetic resonance of rotating solids, Chem. Phys. Lett., 321 (2000) 205-215.

[14] M. H. Levitt, Symmetry in the design of NMR multiple-pulse sequences, J. Chem. Phys., 128 (2008) 052205.

[15] L. Duma, D. Abergel, P. Tekely, G. Bodenhausen, Proton chemical shift anisotropy measurements of hydrogen-bonded functional groups by fast magic angle spinning solid-state NMR spectroscopy, Chem. Commun., (2008) 2361-2363.

[16] E. R. Andrew, A. Bradbury, R. G. Eades, V. T. Wynn, Nuclear cross-relaxation induced by specimen rotation, Phys. Lett., 4 (1963) 99-100.

[17] T. G. Oas, R. G. Griffin, M. H. Levitt, Rotary resonance recoupling of dipolar interactions in solidstate nuclear magnetic resonance spectroscopy, J. Chem. Phys., 89 (1988) 692-695.

[18] Z. Gan, D. M. Grant, R. R. Ernst, NMR chemical shift anisotropy measurements by rf driven rotary resonance, Chem. Phys. Lett., 254 (1996) 349-357.

[19] G. Hou, S. Paramasivam, S. Yan, T. Polenova, A.J. Vega, Multidimensional magic angle spinning NMR spectroscopy for site-resolved measurement of proton chemical shift anisotropy in biological solids, $J$. Am. Chem. Soc., 135 (2013) 1358-1368.

[20] M. Bak, J. T. Rasmussen, N. C. Nielsen, SIMPSON: A general simulation program for solid-state NMR 
spectroscopy, J. Magn. Reson., 147 (2000) 296-330.

[21] S. K. Zaremba, Good lattice points, discrepancy, and numerical integration, Ann. Mat. Pura. Appl. 73, (1966) 293-317.

[22] W. T. Eadie, D. Drijard, F. E. James, M. Roos, B. Sadoulet, Statistical methods in experimental physics, North Holland Publishing Co., Amsterdam, 1971.

[23] A. Brinkmann, A. P. M. Kentgens, Proton-selective ${ }^{17} \mathrm{O}-{ }^{1} \mathrm{H}$ distance measurements in fast magic angle spinning solid-state NMR spectroscopy for the determination of hydrogen bond lengths, J. Am. Chem. Soc., 128 (2006) 14758-14759.

[24] M. N. Frey, T. F. Koetzle, M. S. Lehmann, W. C. Hamilton, Precision neutron diffraction structure determination of protein and nucleic acid components. X. A comparison between the crystal and molecular structures of L-tyrosine and L-tyrosine hydrochloride, J. Chem. Phys., 58 (1973) 2547-2556.

[25] J. P. Glusker, J. A. Minkin, A. L. Patterson, X-ray crystal analysis of the substrates of aconitase. IX. A refinement of the structure of anhydrous citric acid, Acta Crystallogr. B, 25 (1968) 1066-1072.

[26] J. C. C. Chan, R. Tycko, Recoupling of chemical shift anisotropies in solid-state NMR under highspeed magic-angle spinning and in uniformly ${ }^{13}$ C-labeled systems, J. Chem. Phys., 118, (2003) 8378-8389.

[27] Y. Nishiyama, T. Yamazaki, T. Terao, Development of modulated rf sequences for decoupling and recoupling of nuclear-spin interactions in sample-spinning solid-state NMR: application to chemical-shift anisotropy determination. J. Chem. Phys., 124, (2006) 64304-64304.

[28] X. Lu, O. Lafon, J. Trebosc, A. S. L. Thankamony, Y. Nishiyama, Z. Gan, P. K. Madhu, J.-P. Amoureux, Detailed analysis of the TIMES and TIMES0 high-resolution MAS methods for high-resolution proton NMR, J. Magn. Reson., 223, (2012) 219-227. 